\title{
The Selection of Time Interval Between Surgery and Adjuvant Therapy in Early Stage Cervical Cancer
}

\author{
Kai-Yun You, MD, Xin-Hui Zhou, MD, Yan-Hui Jiang, MD, Zhuo-Fei Bi, MD, \\ Yi-Min Liu, MD, and Xing-Sheng Qiu, MD
}

Objectives: The optimal interval between surgery and adjuvant treatment has not yet been found in cervical cancer. And whether patients with different FIGO stage should choose different interval is unknown. The purpose of this study was to evaluate whether interval has a different effect on oncologic outcome for patients with different tumor stages.

Methods: We performed a retrospective study of 226 cervical cancer patients who were treated by surgery and adjuvant therapy from May 2005 to August 2015. All patients were divided into 2 groups according to the interval of 5 weeks. Overall survival (OS) and diseasefree survival (DFS) were compared between patients with interval shorter and longer than 5 weeks in the whole group and subgroups. Recurrence patterns were also analyzed. Multivariate analysis was performed to explore clinical factors significantly associated with DFS, local recurrence-free survival and distant metastasis-free survival for patients with stage IB2-IIA.

Results: For patients with stage IA2-IB1, the 5-year OS and DFS were similar between groups of short and long interval with also the comparable results of local and distant failure. For patients with IB2-IIA, both the OS and DFS in the short-interval group were higher than that in the long-interval group. Besides, the rates of local recurrence were found higher in the group of long interval compared with short interval. Multivariable analysis indicated that time interval was an independent predictor of DFS and local recurrence-free survival for patients with stage IB2-IIA. Conclusions: In cervical cancer patients, time interval between surgery and adjuvant therapy may have different effects on the prognosis in different FIGO stages.

Key Words: Cervical cancer, Radiotherapy, Chemotherapy, Time interval

Received September 7, 2017, and in revised form March 27, 2018.

Accepted for publication May 28, 2018.

(Int J Gynecol Cancer 2018;28: 1325-1332)

$T$ he standard of care for early-stage cervical cancer is surgery, and definitive radiotherapy (RT) serves as an alternative if surgery is not suitable to be administered or is refused by patients. ${ }^{1}$ After surgery, some pathological findings, including metastasis-positive pelvic nodes, surgical margins, and/or positive parametrial involvement $(\mathrm{PI})$ are regarded as indicating a high risk of recurrence, and postoperative chemo-RT is suggested for patients with these findings. ${ }^{2}$ Moreover, patients with tumors that display a combination of intermediate-risk factors, such as large size, lymphovascular space involvement (LVSI), or deep
Department of Radiation Oncology, SunYat-Sen Memorial Hospital, SunYat-Sen University, Guangzhou, China.

Copyright (C) 2018 The Author(s). Published by Wolters Kluwer Health, Inc. on behalf of IGCS and ESGO. This is an open-access article distributed under the terms of the Creative Commons Attribution-Non Commercial-No Derivatives License 4.0 (CCBY-NC-ND), where it is permissible to download and share the work provided it is properly cited. The work cannot be changed in any way or used commercially without permission from the journal. ISSN: $1048-891 \mathrm{X}$

DOI: $10.1097 / \mathrm{IGC} .0000000000001307$
Address correspondence and reprint requests to Yi-Min Liu, MD, Department of Radiation Oncology, SunYat-Sen Memorial Hospital, SunYat-Sen University, Guangzhou, China. E-mail: liuyiminsys@sina.cn and Xing-Sheng Qiu, MD, Department of Radiation Oncology, SunYat-Sen Memorial Hospital, SunYat-Sen University, Guangzhou, China. E-mail: qiuxingsheng@sina.cn.

The authors declare no conflicts of interest.

Kai-Yun You, Xin-Hui Zhou, and Yan-Hui Jiang are equal contributors. 
stromal invasion (DSI), are also considered to be at risk of recurrence and require postoperative pelvic RT. ${ }^{3}$

Currently, adjuvant RT or chemo-RT for early-stage uterine cervical cancer is usually initiated 4 to 6 weeks after radical hysterectomy, allowing time for wound healing. However, there is no robust evidence supporting that, and the optimal interval between surgery and adjuvant therapy has not yet been fully determined. ${ }^{4}$ Besides, various reports have suggested that the time interval (TI) from surgery to adjuvant therapy may have an impact on survival in patients with many types of cancer, including breast, colorectal, endometrial, and head and neck cancers. ${ }^{5-8}$ It is reasonable to consider that this TI may also influence survival in patients with cervical cancer. Thus, we performed our present study with the aim to investigate whether the interval between surgery and adjuvant therapy influences oncologic outcomes, which may help us to optimize individual treatments.

\section{MATERIALS AND METHODS}

\section{Ethics Statement}

This research was approved by the ethics committee of Sun Yat-sen Memorial Hospital, and written informed consent was obtained from every patient included in the study.

\section{Patients and Procedures}

The data were extracted from a prospectively collected database that enrolled all patients who underwent surgical treatment at Sun Yat-sen Memorial Hospital between May 2005 and August 2015. The database included information on patient characteristics, operative findings, pathologic reports, adjuvant treatment, and follow-up data. The selection criteria for the study were as follows: (1) pathologically confirmed uterine cervical cancer; (2) received surgery, followed by adjuvant RT or adjuvant chemo-RT; (3) normal liver and renal function; (4) no evidence of distant metastases during the treatment; and (5) no concurrent malignancy or prior history of RT to the pelvis. Patients with only one intermediate-risk factor were not included because adjuvant treatment for them is controversial in our hospital. Finally, 226 patients met the inclusion criteria and were analyzed in the present study after reviewing the clinical data.

\section{Chemotherapy}

Patients who were classified in the high-risk group were administered with adjuvant chemotherapy. A regimen containing paclitaxel and cisplatin was administered to the patients every 3 weeks. Details of the dose are as follows: paclitaxel, $135 \mathrm{mg} / \mathrm{m}^{2}$ over 3-h IV on day 1; and cisplatin, $70 \mathrm{mg} / \mathrm{m}^{2}, 2$-h IV infusion on day 1 . The median number of administered cycles in patients who received chemotherapy was 3 (2-4). In addition, patients within the intermediate-risk group were recommended to undergo adjuvant RT alone and chemotherapy was not administered to each of them.

\section{External Beam Radiotherapy}

All the patients received a standard protocol of postoperative radiation with three-dimensional conformal RT. The prescribed dose to the whole pelvis was 45 to $50 \mathrm{~Gy}$, which was delivered in 1.8- to 2.0-Gy fractions once daily for 5 days per week. The clinical target volume included the primary tumor bed, supravaginal portion, paracervical tissue, common iliac lymph nodes, internal and external iliac lymph nodes, obturator lymph nodes, and sacral lymph nodes. Roughly, the superior border of the clinical target volume was the bottom of L4, and the inferior border was the lower margin of the obturator. The anterior border was the posterior margin of the bladder. When lateral fields were used, the posterior border encompassed S2.

\section{Follow-Up Evaluation}

In our hospital, patients were recommended to be evaluated every 3 months for the first 2 years, every 6 months during the following 3 years, and annually thereafter. Complete

TABLE 1A. Patient demographics, baseline tumor characteristics, type of surgery, and pathologic outcome

\begin{tabular}{|c|c|c|c|}
\hline Variable & $\begin{array}{c}\text { Interval } \leq \\
5 \text { wk }(n=104)\end{array}$ & $\begin{array}{c}\text { Interval }> \\
5 \mathrm{wk}(\mathrm{n}=122)\end{array}$ & $P$ \\
\hline Age, y & & & 0.041 \\
\hline Median & 54 & 57 & \\
\hline Stage & & & 0.644 \\
\hline IA2 & $3(2.9 \%)$ & $4(3.3 \%)$ & \\
\hline IB1 & $56(53.8 \%)$ & $71(58.2 \%)$ & \\
\hline IB2 & $43(41.4 \%)$ & $42(34.4 \%)$ & \\
\hline IIA & $2(1.9 \%)$ & $5(4.1 \%)$ & \\
\hline Histology & & & 0.492 \\
\hline SCC & $62(59.6 \%)$ & $82(67.2 \%)$ & \\
\hline $\mathrm{AC}$ & $31(29.8 \%)$ & $30(24.6 \%)$ & \\
\hline ASC & $11(10.6 \%)$ & $10(8.2 \%)$ & \\
\hline LVSI & & & 0.506 \\
\hline Yes & $60(57.7 \%)$ & $65(53.3 \%)$ & \\
\hline No & $44(42.3 \%)$ & $57(46.7 \%)$ & \\
\hline DSI & & & 0.292 \\
\hline Yes & $62(59.6 \%)$ & $81(66.4 \%)$ & \\
\hline No & $42(40.4 \%)$ & $41(33.6 \%)$ & \\
\hline $\begin{array}{l}\text { Lymph node } \\
\text { metastasis }\end{array}$ & & & 0.639 \\
\hline Yes & $22(21.2 \%)$ & $29(23.8 \%)$ & \\
\hline No & $82(78.8 \%)$ & $93(76.2 \%)$ & \\
\hline PI & & & 0.573 \\
\hline Yes & $28(26.9 \%)$ & $37(30.3 \%)$ & \\
\hline No & $76(73.1 \%)$ & $85(69.7 \%)$ & \\
\hline Vaginal involvement & & & 0.534 \\
\hline Yes & $26(25.0 \%)$ & $35(28.7 \%)$ & \\
\hline No & $78(75.0 \%)$ & $87(71.3 \%)$ & \\
\hline Adjuvant therapy & & & 0.477 \\
\hline RT & $42(40.4 \%)$ & $55(45.1 \%)$ & \\
\hline Chemo-RT & $62(59.6 \%)$ & $67(54.9 \%)$ & \\
\hline
\end{tabular}


TABLE 1B. Correlation between the different features investigated and the survival rates for the whole group

\begin{tabular}{|c|c|c|c|c|c|}
\hline Variable & No. Patients (\%) & 5-y DFS & $P$ & 5-y OS & $\boldsymbol{P}$ \\
\hline Age, y & & & 0.188 & & 0.876 \\
\hline$\leq 55$ & $108(46.9 \%)$ & $82.33 \%$ & & $88.39 \%$ & \\
\hline$>55$ & $118(53.1 \%)$ & $74.25 \%$ & & $83.64 \%$ & \\
\hline Stage & & & 0.161 & & 0.173 \\
\hline IA2 & $7(3.1 \%)$ & $85.71 \%$ & & $85.71 \%$ & \\
\hline IB1 & $127(56.2 \%)$ & $83.79 \%$ & & $90.87 \%$ & \\
\hline IB2 & $85(37.6 \%)$ & $70.14 \%$ & & $82.54 \%$ & \\
\hline IIA & $7(3.1 \%)$ & $53.57 \%$ & & $64.29 \%$ & \\
\hline Histology & & & 0.498 & & 0.908 \\
\hline $\mathrm{SCC}$ & $144(63.7 \%)$ & $80.44 \%$ & & $88.17 \%$ & \\
\hline $\mathrm{AC}$ & $61(27.0 \%)$ & $72.50 \%$ & & $86.86 \%$ & \\
\hline $\mathrm{ASC}$ & $21(9.3 \%)$ & $80.95 \%$ & & $85.71 \%$ & \\
\hline LVSI & & & 0.833 & & 0.947 \\
\hline Yes & $125(55.3 \%)$ & $77.38 \%$ & & $85.84 \%$ & \\
\hline No & $101(44.7 \%)$ & $79.30 \%$ & & $86.51 \%$ & \\
\hline DSI & & & 0.041 & & 0.058 \\
\hline Yes & $143(63.3 \%)$ & $74.06 \%$ & & $82.52 \%$ & \\
\hline No & $83(36.7 \%)$ & $86.65 \%$ & & $93.79 \%$ & \\
\hline Lymph node metastasis & & & 0.030 & & 0.026 \\
\hline Yes & $51(22.6 \%)$ & $66.58 \%$ & & $79.99 \%$ & \\
\hline No & $175(77.4 \%)$ & $82.40 \%$ & & $88.09 \%$ & \\
\hline PI & & & 0.003 & & 0.048 \\
\hline Yes & $65(28.8 \%)$ & $62.41 \%$ & & $80.79 \%$ & \\
\hline No & $161(71.2 \%)$ & $85.02 \%$ & & $87.91 \%$ & \\
\hline Vaginal involvement & & & 0.067 & & 0.095 \\
\hline Yes & $61(27.0 \%)$ & $73.57 \%$ & & $81.81 \%$ & \\
\hline No & $165(73.0 \%)$ & $81.64 \%$ & & $89.36 \%$ & \\
\hline Risk group & & & 0.033 & & 0.528 \\
\hline Intermediate-risk group & $97(42.9 \%)$ & $87.21 \%$ & & $89.96 \%$ & \\
\hline High-risk group & $127(57.1 \%)$ & $71.84 \%$ & & $83.64 \%$ & \\
\hline
\end{tabular}

blood cell counts, biochemical routines, squamous cell carcinoma (SCC) antigen, and physical examinations were the routine evaluations during each visit. Vaginal cytology assessments were also performed for the detection of lower genital tract neoplasia. Chest radiography and computed tomography or magnetic resonance imaging of the abdomen and pelvis were conducted every 6 months with the aim of detecting possible recurrent disease. If recurrent signs were suspected in a patient, biopsy was performed whenever possible. Disease-free survival (DFS) and overall survival (OS) were defined from the date of diagnosis to the date of recurrence or metastasis and to the date of death, respectively. For surviving patients, they were defined to the date of the last follow-up.

\section{Statistical Analysis}

All statistical analyses were performed using SPSS software, version 19.0. Categorical variables were analyzed 
TABLE 2. Survival for the whole group of patients

\begin{tabular}{|c|c|c|c|c|c|}
\hline \multirow[b]{2}{*}{ Group } & \multicolumn{2}{|c|}{$\begin{array}{c}\text { Interval } \leq \\
5 \text { wk }(n=104)\end{array}$} & \multicolumn{2}{|c|}{$\begin{array}{c}\text { Interval }> \\
5 \text { wk }(n=122)\end{array}$} & \multirow[b]{2}{*}{$\boldsymbol{P}$} \\
\hline & $3-y$ & $5-y$ & $3-y$ & $5-y$ & \\
\hline OS & $90.65 \%$ & $86.70 \%$ & $80.85 \%$ & $77.74 \%$ & $0.105^{*}$ \\
\hline DFS & $88.06 \%$ & $82.61 \%$ & $83.68 \%$ & $73.68 \%$ & $0.322 *$ \\
\hline
\end{tabular}

using the $\chi^{2}$ test or Fisher exact test. Continuous variables were analyzed using the Student $t$ test or the Mann-Whitney $U$ test. The Kaplan-Meier method was used to compare DFS and OS rates. Multivariate analysis of DFS, local recurrencefree survival (LRFS), and distant metastasis-free survival (DMFS) were performed using Cox proportional hazards regression, and the Cox proportional hazards model was performed using a forward conditional selection of variables. Variables with $P$ values less than 0.2 were entered into the Cox model. $P$ value of less than 0.05 was considered to be statistically significant.

\section{RESULTS}

\section{Clinical Characteristics}

In total, 226 patients were enrolled in our present study. The median interval time between surgery and adjuvant therapy was roughly 5 weeks (2-8 weeks); this was chosen as the cut-off value to separate all the patients into 2 groups. A total of 104 patents received adjuvant therapy less than 5 weeks after surgery, whereas the remaining 122 patents received adjuvant therapy more than 5 weeks after surgery. Compared with patients with longer intervals between adjuvant therapy and surgery, those with short intervals presented no differences in histology, tumor stage, LVSI, DSI, PI, lymph node metastasis, vaginal involvement, and adjuvant therapy. However, patients in the long interval group were significantly older than those in the short interval group (Table 1a).

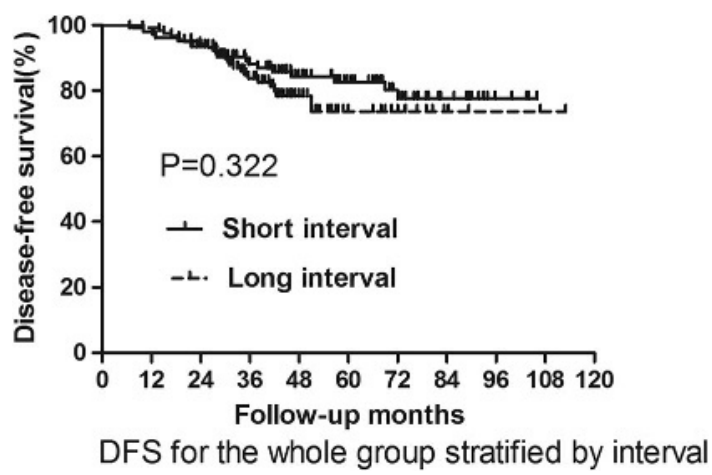

FIGURE 2. DFS for the whole group stratified by interval. No significant difference was found in DFS between patients with short and long interval for whole group $(P=0.322)$.

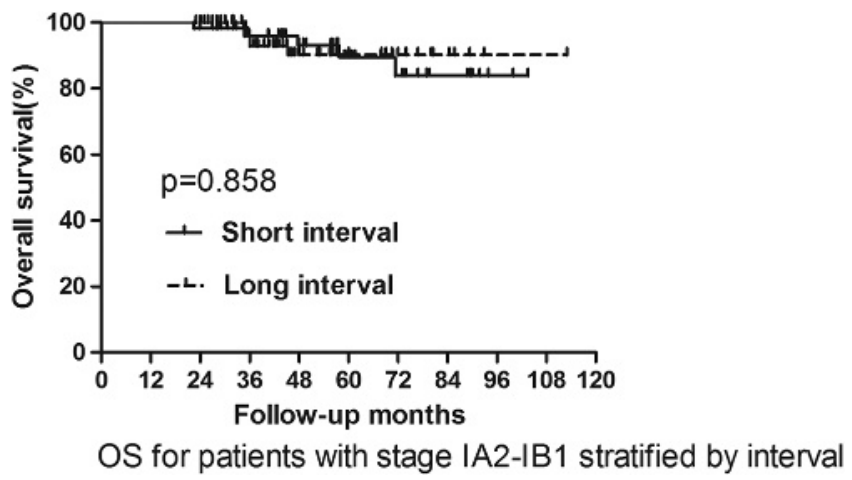

FIGURE 3. OS for patients with stage IA2-IB1 stratified by interval. No significant difference was found in OS between patients with short and long interval for the subgroup $(P=0.858)$.

\section{Survival Analysis for All Patients}

Among all patients, 24 died during follow-up. On univariate analysis, the clinical factors associated with decreased OS were lymph node metastasis $(P=0.026)$ and PI $(P=0.048)$ (Table 1b). And the 5-year OS rates in the short and long interval groups were $86.70 \%$ and $77.74 \%$, respectively (Fig. 1, Table 2). No significant difference was found in the 5-year OS between the 2 groups $(P=0.105)$. Recurrence was found in 42 patients. Among them, 10 patients developed local recurrence and 26 patients presented with distant metastasis. Besides, 6 patients developed both local and distant failure. The clinical factors correlated with worse DFS on univariate analysis were positive parametrial invasion, lymph node metastasis, DSI, and highrisk group (Table 1b). As for the clinical factor of TI, the 5 -year DFS rates in the short and long interval groups were $82.61 \%$ and $73.68 \%$, with no significant difference between the 2 groups $(P=0.322)$ (Fig. 2, Table 2).

\section{Survival Analysis for the Subgroups}

For patients with stage IA2 to IB1 cervical cancer, 10 died during follow-up and 19 developed recurrence. Three patients only recurred locally and 14 patients only developed

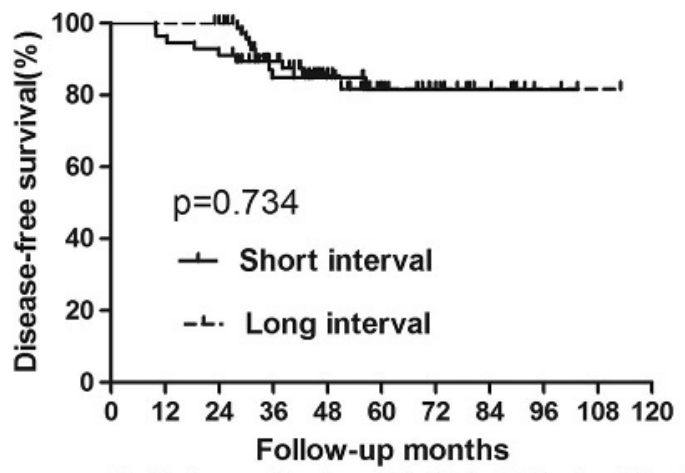

DFS for patients with IA2-IB1 stratified by interval

FIGURE 4. DFS for patients with stage IA2-IB1 stratified by interval. No significant difference was found in DFS between patients with short and long interval for the subgroup $(P=0.734)$. 
TABLE 3. Survival for patients with stage IA2-IB1

\begin{tabular}{|c|c|c|c|c|c|}
\hline \multirow[b]{2}{*}{ Group } & \multicolumn{2}{|c|}{$\begin{array}{c}\text { Interval } \leq \\
5 \text { wk }(n=56)\end{array}$} & \multicolumn{2}{|c|}{$\begin{array}{c}\text { Interval }> \\
5 \mathrm{wk}(\mathrm{n}=\mathbf{7 8})\end{array}$} & \multirow[b]{2}{*}{$P$} \\
\hline & $3-y r$ & $5-y r$ & $3-y r$ & $5-y r$ & \\
\hline OS & $95.88 \%$ & $89.48 \%$ & $92.84 \%$ & $90.20 \%$ & $0.858^{*}$ \\
\hline DFS & $84.79 \%$ & $81.53 \%$ & $89.37 \%$ & $81.63 \%$ & $0.734 *$ \\
\hline
\end{tabular}

distant metastasis. In addition, 2 patients presented with both local and distant metastases. The 5-year OS and DFS rates in the short and long interval groups were $89.48 \%$ and $90.20 \%$ and $81.53 \%$ and $81.63 \%$, respectively (Figs. 3, 4, Table 3 ). No significant differences were found in both OS and DFS rates between the 2 groups ( $P=0.858$ and $P=0.734$, respectively). The recurrence pattern was further analyzed with results showing that there were no differences in both the local and distant failure rates between patients in the short and long interval groups (Table 4).

For patients with stage IB2 to IIA cervical cancer, 23 developed recurrence, of which 14 died because of tumor recurrence. Specifically, 7 patients developed only local recurrence and 12 presented with only distant metastasis. Four patients developed both local and distant metastases. The 5-year OS rate in the short interval group was significantly greater than that in the long interval group $(89.20 \%$ vs $72.14 \%, P=0.050)$ (Fig. 5, Table 5). As for the 5-year DFS rates, it was also found that patients in the short interval group acquired better DFS compared with those in the long interval group $(83.77 \%$ vs $59.65 \%, P=0.034$ ) (Fig. 6, Table 5). Deeper analysis was performed on recurrence patterns, and we found that the rate of local recurrence was significantly higher in the long interval group than that in the short interval group. Meanwhile, no difference was shown in the distant metastasis between the 2 groups (Table 6).

\section{Clinical Predictors for DFS, LRFS, and DMFS in Patients With Stage IB2 to IIA Cervical Cancer}

In multivariable analysis, both the TI and lymph node metastasis were independent predictors of DFS in patients with stage IB2 to IIA cervical cancer. In additions, the TI was especially associated with LRFS, although it did not predict

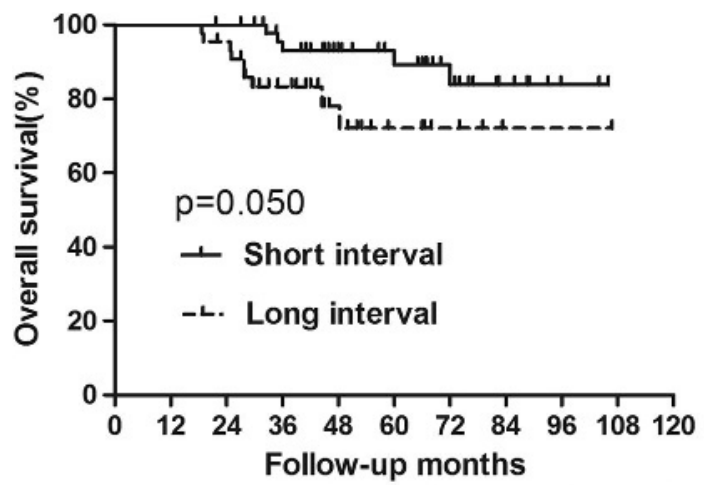

OS for patients with IB2-IIA stratified by interval

FIGURE 5. OS for patients with stage IB2-IIA stratified by interval. No significant difference was found in OS between patients with short and long interval for the subgroup $(P=0.050)$.

DMFS significantly. Besides, PI also predicted poor LRFS, and lymph node metastasis was significantly correlated with impaired DMFS (Table 7).

\section{CONCLUSIONS}

In our present study, we demonstrated that the TI between surgery and adjuvant RT (chemo-RT) may have different effects on oncologic outcomes in patients with cervical cancer of different International Federation of Gynecology and Obstetrics (FIGO) stages. In detail, for patients with stage IB2 to IIA cervical cancer, the patients in the long interval group achieved poorer DFS than those in the short interval group. However, in patients with stage IA2 to IB1 cervical cancer, no significant differences were found in DFS and OS between patients in the long and short interval groups. Further analysis was performed on the recurrence patterns in patients with stage IB2 to IIA cervical cancer, and we found that long intervals resulted in poorer local control, thus leading to impaired DFS and OS. Multivariable analysis was also performed, which showed that long intervals significantly predicted poorer LRFS. A possible explanation is that the micrometastases or residual cancer cells may proliferate during the interval from surgery to adjuvant therapy and the increased time may have offered the chance for increased proliferation. ${ }^{9,10}$ It has been reported that surgery may lead to early accelerated growth of micrometastases and the conversion of dormant tumor cells into

TABLE 4. Recurrence patterns for patients with stage IA2-IB1

\begin{tabular}{|c|c|c|c|c|c|}
\hline \multirow[b]{2}{*}{ Group } & \multicolumn{2}{|c|}{ Interval $\leq 5$ wk $(n=56)$} & \multicolumn{2}{|c|}{ Interval $>5$ wk $(\mathrm{n}=78)$} & \multirow[b]{2}{*}{$P$} \\
\hline & $3-\mathbf{y}$ & $5-\mathbf{y}$ & $3-\mathbf{y}$ & $5-\mathbf{y}$ & \\
\hline LR & $2(3.6 \%)$ & $2(3.6 \%)$ & $3(3.8 \%)$ & $3(3.8 \%)$ & $0.951^{*}$ \\
\hline SM & $6(10.7 \%)$ & $8(14.3 \%)$ & $4(5.1 \%)$ & $7(9.0 \%)$ & $0.637^{*}$ \\
\hline
\end{tabular}


TABLE 5. Survival for the patients with stage IB2-IIA

\begin{tabular}{|c|c|c|c|c|c|}
\hline \multirow[b]{2}{*}{ Group } & \multicolumn{2}{|c|}{$\begin{array}{c}\text { Interval } \leq \\
5 \text { wk }(n=48)\end{array}$} & \multicolumn{2}{|c|}{$\begin{array}{c}\text { Interval }> \\
5 \text { wk }(n=44)\end{array}$} & \multirow[b]{2}{*}{$\boldsymbol{P}$} \\
\hline & $3-y$ & $5-y$ & $3-y$ & $5-y$ & \\
\hline OS & $93.07 \%$ & $89.20 \%$ & $83.36 \%$ & $72.14 \%$ & $0.050^{*}$ \\
\hline DFS & $91.53 \%$ & $83.77 \%$ & $73.94 \%$ & $59.65 \%$ & $0.034 *$ \\
\hline
\end{tabular}

rapidly proliferating ones. ${ }^{11}$ Besides, long intervals between the surgical removal of primary tumors and administration of chemotherapy may weaken the cytotoxic effects of the chemotherapeutic agents, leading to a decrease in the proliferative index of tumor cells. ${ }^{12}$ Thus, it seems rational to initiate the administration of adjuvant treatments after surgery as soon as possible if the patients' general condition permits.

Currently, few studies have investigated the impact of TIs between surgery and adjuvant treatment on the prognosis of cervical cancer. In a study performed by Hanprasertpong et al, 110 patients with stage IA2 to IB1 cervical cancer who underwent radical resection and adjuvant therapy were included, and they were divided into 2 groups based on the cutoff TIs of 4 and 6 weeks, respectively. The results showed that, according to the TI ( $\leq 4 \mathrm{vs}>4$ weeks, and $\leq 6 \mathrm{vs}>6$ weeks), no statistical differences were found in 5-year recurrence-free survival (RFS) $(89.2 \%$ vs $81.0 \%$, and $83.2 \%$ vs $100.0 \%$, respectively) or 5 -year OS rates (90.9\% vs $97.2 \%$, and $93.2 \%$ vs $100.0 \%$, respectively). However, it was found that the delay in initiating adjuvant therapy after surgery beyond 4 weeks might result in poorer RFS in patients with SCC, whereas patients with adenocarcinoma (AC) or adenosquamous cell carcinoma (ASC) did not show any detriments from delayed administration of adjuvant therapy. ${ }^{4}$ In another study conducted by Kim et al, 98 patients with pelvic lymph node metastasis, positive resection margins, and/or parametrial invasion who received adjuvant chemo-RTwere included. The first cycle of platinum-based adjuvant chemotherapy was initiated within

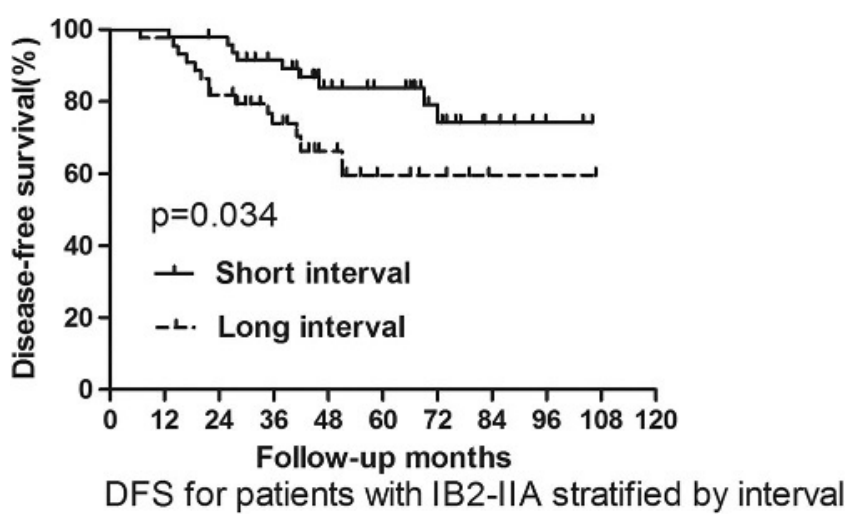

FIGURE 6. DFS for patients with stage IB2-IIA stratified by interval. No significant difference was found in DFS between patients with short and long interval for the subgroup $(P=0.034)$.
2 to 3 weeks after surgery (median, 12 days) and continued every 4 weeks for a total of 4 cycles. Adjuvant RTwas performed during the second and third cycles of chemotherapy. Their results showed that the oncologic outcomes were quite favorable with 5 -year RFS and cancer-specific survival rates of $87.6 \%$ and $90.6 \%$, respectively. ${ }^{13}$ Besides, Jhawar et al performed an analysis based on data including 3051 nonmetastatic cervical cancer patients undergoing hysterectomy followed by adjuvant chemoradiation. Their results also indicated that a longer interval between surgery and adjuvant chemoradiation leads to impaired survival. Thus, they suggested a TI (surgery to adjuvant chemoradiation) of less than 8 weeks should be attempted whenever clinically feasible. ${ }^{14}$ In our present study, the 5-year OS and DFS rates were slightly lower than those discussed earlier; this was due to patients with stage IB2 and IIA cervical cancer also being included in the analysis. The new finding in our report was that we discovered the TI that may impart different effects in patients with cervical cancer of different FIGO stages.

The TI from surgery to adjuvant therapy can have an impact in other types of cancer. It was reported that a delay in RT of more than 9 weeks was an independent significant predictor for poor disease-specific survival $(P<0.005)$ in patients with endometrial cancer who received postoperative RT. ${ }^{15}$ In a study by Klein et al, 1827 patients with stage III colonic cancer were included; their results showed that adjuvant therapy that was initiated within 4 and 8 weeks improved survival when compared with initiating adjuvant therapy later than 8 weeks (hazard ratio [95\% confidence interval]: 1.7 [1.1-2.6]; $P=0.024$ and 1.4 [1.07-1.8]; $P=0.013$, respectively). ${ }^{9}$ However, several other studies have found that TI is not associated with clinical outcomes in patients with breast cancer, soft tissue sarcoma, and gastric cancer. ${ }^{16-18}$ This simply indicates that the TI may have different impacts on survival in different cancers at different stages, and some intrinsic factors, although not fully determined, may play a role.

When adjuvant therapy was administered earlier, the possible increase in treatment-related toxicities should be considered because of inadequate recovery from surgery. Kim et al reported that the incidences of grade 3 to 4 hematologic and gastrointestinal toxicities were $37.8 \%$ and $14.3 \%$, respectively, in patients who received adjuvant treatment within 2 to 3 weeks after surgery. ${ }^{13}$ Moreover, they suggested that early administration of adjuvant concurrent chemo-RT was safe

TABLE 6. Recurrence patterns for patients with stage IB2-IIA

\begin{tabular}{|c|c|c|c|c|c|}
\hline \multirow[b]{2}{*}{ Group } & \multicolumn{2}{|c|}{$\begin{array}{c}\text { Interval } \leq \\
5 \text { wk }(n=48)\end{array}$} & \multicolumn{2}{|c|}{$\begin{array}{c}\text { Interval > } \\
5 \text { wk }(\mathrm{n}=44)\end{array}$} & \multirow[b]{2}{*}{$\boldsymbol{P}$} \\
\hline & $3-y$ & $5-y$ & $3-y$ & $5-y$ & \\
\hline LR & $1(2.1 \%)$ & $2(4.2 \%)$ & $5(11.4 \%)$ & $8(18.2 \%)$ & $0.021 *$ \\
\hline SM & $3(6.3 \%)$ & $6(12.5 \%)$ & $8(18.2 \%)$ & $9(20.5 \%)$ & $0.187 *$ \\
\hline
\end{tabular}


TABLE 7. Multivariate analyses of DFS, LRFS, and DMFS for patients with stage IB2-IIA

\begin{tabular}{|c|c|c|c|c|c|c|}
\hline \multirow[b]{2}{*}{ Variable } & \multicolumn{2}{|l|}{ DFS } & \multicolumn{2}{|l|}{ LRFS } & \multicolumn{2}{|l|}{ DMFS } \\
\hline & HR (95\% CI) & $P$ & HR (95\% CI) & $\boldsymbol{P}$ & HR (95\% CI) & $\boldsymbol{P}$ \\
\hline \multicolumn{7}{|l|}{ TI } \\
\hline$\leq 5 \mathrm{wk}$ vs $>5 \mathrm{wk}$ & $0.325(0.145-0.879)$ & 0.027 & $0.138(0.015-0.963)$ & 0.042 & $0.434(0.168-1.095)$ & 0.064 \\
\hline \multicolumn{7}{|c|}{ Lymph node metastasis } \\
\hline No vs yes & $0.216(0.117-0.850)$ & 0.018 & $0.211(0.121-1.083)$ & 0.055 & $0.309(0.116-0.963)$ & 0.042 \\
\hline \multicolumn{7}{|l|}{ PI } \\
\hline No vs yes & $0.556(0.280-1.106)$ & 0.095 & $0.209(0.042-0.972)$ & 0.047 & $0.703(0.304-1.449)$ & 0.516 \\
\hline \multicolumn{7}{|l|}{ Age } \\
\hline$>55$ vs $\leq 55$ & $1.420(0.738-2.733)$ & 0.293 & $0.797(0.267-2.381)$ & 0.685 & $1.522(0.710-3.261)$ & 0.303 \\
\hline \multicolumn{7}{|l|}{ LVSI } \\
\hline Yes vs no & $1.108(0.551-2.230)$ & 0.773 & $0.926(0.297-2.890)$ & 0.895 & $1.497(0.649-3.452)$ & 0.344 \\
\hline \multicolumn{7}{|l|}{ DSI } \\
\hline Yes vs no & $2.065(0.954-4.474)$ & 0.066 & $3.531(0.932-6.530)$ & 0.053 & $1.390(0.615-3.141)$ & 0.429 \\
\hline \multicolumn{7}{|l|}{ Vaginal involvement } \\
\hline Yes vs no & $2.011(0.860-3.413)$ & 0.072 & $3.247(0.862-5.768)$ & 0.057 & $1.416(0.637-3.275)$ & 0.387 \\
\hline \multicolumn{7}{|l|}{ Histology } \\
\hline $\mathrm{SCC}$ vs AC & $0.685(0.229-2.047)$ & 0.498 & $0.239(0.056-1.025)$ & 0.058 & $1.126(0.252-5.037)$ & 0.876 \\
\hline ASC vs AC & $0.945(0.301-2.965)$ & 0.923 & $0.394(0.082-1.316)$ & 0.070 & $1.590(0.345-7.328)$ & 0.288 \\
\hline
\end{tabular}

because the incidence of severe toxicities (grade 3-4) was comparable with that in patients with longer interval times. ${ }^{19-21}$ In our present study, the incidence of severe diarrhea was higher in patients with short intervals than in those with long intervals, whereas other types of toxicities were similar between the 2 groups (Table 8).

There were some limitations, such as the short follow-up and retrospective design in our study. Another important limitation was that the small sample size in the different subgroups may have affected the reality of the results to some extent. Although we have suggested that a longer interval may lead to impaired survival for patients with stage IB2 to IIA cervical cancer, the optimal interval for the patients still needs to be investigated. A large study including sufficient cases might address this question.

TABLE 8. Severe toxicity (Grade $\geq 3$ ) of adjuvant therapy

\begin{tabular}{lccc}
\hline $\begin{array}{l}\text { Toxicity Type } \\
(\text { Grade } \geq \mathbf{3})\end{array}$ & $\begin{array}{c}\text { Interval } \leq \\
\mathbf{5} \text { wk }(\mathbf{n}=\mathbf{1 0 4})\end{array}$ & $\begin{array}{c}\text { Interval }> \\
\mathbf{5} \mathbf{w k}(\mathbf{n}=\mathbf{1 2 2})\end{array}$ & $\boldsymbol{P}$ \\
\hline Abdominal pain & & & \\
Nausea & $4(3.8 \%)$ & $4(3.3 \%)$ & 0.818 \\
Vomiting & $3(2.9 \%)$ & $3(2.5 \%)$ & 0.843 \\
Enteritis & $2(1.9 \%)$ & $2(1.6 \%)$ & 0.872 \\
Diarrhea & $16(15.4 \%)$ & $9(7.4 \%)$ & 0.056 \\
Constipation & $2(1.9 \%)$ & $2(1.6 \%)$ & 0.872 \\
Hemoglobin & $3(2.9 \%)$ & $2(1.6 \%)$ & 0.526 \\
Platelets & $1 .(1.0 \%)$ & $2(1.6 \%)$ & 0.657 \\
\hline
\end{tabular}

In conclusion, our study indicated that the FIGO stage should be considered when deciding when to administer adjuvant therapy for patients with cervical cancer after surgery. Although we suggested that long intervals may result in poorer survival for patients with stage IB2 to IIA cervical cancer, further larger-scale cohort studies are still warranted to confirm our results. We strongly suggest the tailored selection of TIs for different patients.

\section{REFERENCES}

1. Landoni F, Maneo A, Colombo A, et al. Randomised study of radical surgery versus radiotherapy for stage Ib-IIa cervical cancer. Lancet. 1997;350:535-540.

2. Peters WA 3rd, Liu PY, Barrett RJ 2nd, et al. Concurrent chemotherapy and pelvic radiation therapy compared with pelvic radiation therapy alone as adjuvant therapy after radical surgery in high-risk early-stage cancer of the cervix. J Clin Oncol. 2000;18:1606-1613.

3. Rotman M, Sedlis A, Piedmonte MR. A phase III randomized trial of postoperative pelvic irradiation in Stage IB cervical carcinoma with poor prognostic features: follow-up of a gynecologic oncology group study. Int J Radiat Oncol Biol Phys. 2006;65:169-176.

4. Hanprasertpong J, Jiamset I, Geater A, et al. Impact of time interval between radical hysterectomy with pelvic node dissection and initial adjuvant therapy on oncological outcomes of early stage cervical cancer. J Gynecol Oncol. 2017;28:e42.

5. Biagi JJ, Raphael MJ, Mackillop WJ, et al. Association between time to initiation of adjuvant chemotherapy and survival in colorectal cancer: a systematic review and meta-analysis. JAMA. 2011;305:2335-2342. 
6. Benk V, Joseph L, Fortin P, et al. Effect of delay in initiating radiotherapy for patients with early stage breast cancer. Clin Oncol (R Coll Radiol). 2004;16:6-11.

7. Tribius S, Donner J, Pazdyka H, et al. Survival and overall treatment time after postoperative radio(chemo)therapy in patients with head and neck cancer. Head Neck. 2016;38:1058-1065.

8. Ahmad NR, Lanciano RM, Corn BW, et al. Postoperative radiation therapy for surgically staged endometrial cancer: impact of time factors (overall treatment time and surgery-to-radiation interval) on outcome. Int J Radiat Oncol Biol Phys. 1995;33:837-842.

9. Klein M, Azaquoun N, Jensen BV, et al. Improved survival with early adjuvant chemotherapy after colonic resection for stage III colonic cancer: a nationwide study. J Surg Oncol. 2015;112:538-543.

10. Veenhof AA, Sietses C, von Blomberg BM, et al. The surgical stress response and postoperative immune function after laparoscopic or conventional total mesorectal excision in rectal cancer: a randomized trial. Int J Colorectal Dis. 2011;26:53-59.

11. Gunduz N, Fisher B, Saffer EA. Effect of surgical removal on the growth and kinetics of residual tumor. Cancer Res. 1979;39:3861-3865.

12. Fisher B, Gunduz N, Saffer EA. Influence of the interval between primary tumor removal and chemotherapy on kinetics and growth of metastases. Cancer Res. 1983;43:1488-1492.

13. Kim SW, Chun M, Ryu HS, et al. Long-term results of early adjuvant concurrent chemoradiotherapy for high-risk, early stage uterine cervical cancer patients after radical hysterectomy. BMC Cancer. 2017;17:297.

14. Jhawar S, Hathout L, Elshaikh MA, et al. Adjuvant chemoradiation therapy for cervical cancer and effect of timing and duration on treatment outcome. Int J Radiat Oncol Biol Phys. 2017;98:1132-1141.

15. Cattaneo R 2nd, Hanna RK, Jacobsen G, et al. Interval between hysterectomy and start of radiation treatment is predictive of recurrence in patients with endometrial carcinoma. Int $J$ Radiat Oncol Biol Phys. 2014;88:866-871.

16. Cèfaro GA, Genovesi D, Marchese R, et al. The effect of delaying adjuvant radiation treatment after conservative surgery for early breast cancer. Breast J. 2007;13:575-580.

17. Fourquet J, Sunyach MP, Vilotte F, et al. Time interval between surgery and start of adjuvant radiotherapy in patients with soft tissue sarcoma: a retrospective analysis of 1131 cases from the French Sarcoma Group. Radiother Oncol. 2016;120:156-162.

18. Greenleaf EK, Kulaylat AN, Hollenbeak CS, et al. Timing of adjuvant chemotherapy and impact on survival for resected gastric cancer. Ann Surg Oncol. 2016;23:4203-4213.

19. Sehouli J, Runnebaum IB, Fotopoulou C, et al. A randomized phase III adjuvant study in high-risk cervical cancer: simultaneous radiochemotherapy with cisplatin (S-RC) versus systemic paclitaxel and carboplatin followed by percutaneous radiation (PC-R): a NOGGO-AGO Intergroup Study. Ann Oncol. 2012;23:2259-2264.

20. Lee TS, Kang SB, Kim YT, et al. Chemoradiation with paclitaxel and carboplatin in high-risk cervical cancer patients after radical hysterectomy: a Korean Gynecologic Oncology Group study. Int J Radiat Oncol Biol Phys. 2013;86:304-310.

21. Trifiletti DM, Swisher-McClure S, Showalter TN, et al. Postoperative chemoradiation therapy in high-risk cervical cancer: re-evaluating the findings of Gynecologic Oncology Group Study 109 in a large, population-based cohort. Int $J$ Radiat Oncol Biol Phys. 2015;93:1032-1044. 